\title{
Variability of The Old Nova V603 Aquilae in UV and X-ray bands
}

\author{
W. Borczyk ${ }^{1}$, A. Schwarzenberg-Czerny ${ }^{1,2}$, and P. Szkody ${ }^{3}$ \\ 1 Astronomical Observatory of Adam Mickiewicz University, Sloneczna 36, 60-286 Poznan, Poland \\ 2 Nicolaus Copernicus Astronomical Center, Bartycka 18, 00-716 Warsaw, Poland \\ e-mail: alex@camk.edu.pl \\ 3 Department of Astronomy, University of Washington, Seattle, WA 98195, USA \\ e-mail: szkody@astro.washington.edu
}

Received 5 July 2002 / Accepted 7 March 2003

\begin{abstract}
We present the results of a campaign of simultaneous observations of V603 Aql in the X-ray and UV bands accomplished with the IUE and ROSAT satellites. Simultaneous observations cover 20 orbital cycles, while the total span of all the observations is 40 cycles. This constitutes the longest continuous UV time series of simultaneous space observations of a cataclysmic variable. As all high energy radiation is ultimately powered by accretion, it is perhaps remarkable that we observe some signs of correlations of the UV line and continuum emission with the X-ray flux at zero lag, but none over a range of time lags from 400-40000 s. A hint of some lagged correlation was revealed for UV color and X-ray flux. We detect a very strong modulation of intensity of the UV lines of CIV and SiIV. The modulation appears coherent over the range of observation and its period of 0.1380(5) d is consistent with the spectroscopic orbital period but differs significantly from the period of photometric modulation. Furthermore, we observe variable P-Cygni profiles of the CIV1550 line. Its absorption and emission components are modulated with the orbital period of the binary. These data present evidence for sustained asymmetry of the supersonic wind excited from the accretion disc yet pose difficulties for the current disc and wind models. Since no periodic modulation was found in the X-ray band nor in the UV continuum, the present data do not lend support to our earlier classification of V603 Aql as an intermediate polar.
\end{abstract}

Key words. accretion, accretion disks - stars: individual: V603 Aql - novae, cataclysmic variables - stars: winds, outflows ultraviolet: stars - techniques: image processing

\section{Introduction}

V603 Aquilae made its debut in the annals of astronomy as Nova Aquilae 1918, the brightest Nova of the 20th century. Although it eventually settled into one of the brightest cataclysmic variable $(\mathrm{CV})$ stars $(V \approx 12 \mathrm{mag}$ ), it remained neglected for a long time. In his pioneering survey of the radial velocities of CVs, Kraft (1964) obtained observations of V603 Aql on two consecutive nights and found an approximate orbital period of $0.1382 \mathrm{~d}$. Only recently has this value been refined by Patterson et al. (1997, hereafter P97) to 0.13809(12). Thanks to the unique prism spectrum revealed by Seitter (1990) we know that 20 years before its outburst, V603 Aql did not differ spectroscopically from its current state, at least at low resolution.

Modulation of the light of V603 Aql was found by Drehsel et al. (1981). However it remained for Haefner \& Metz (1985) to demonstrate that the period of modulation was $0.1449 \mathrm{~d}$, definitely in excess of the orbital period. Such peculiar behavior was previously discovered only in one other star, the nova-like TT Ari. This excess in period between the

Send offprint requests to: A. Schwarzenberg-Czerny, e-mail: bori@moon. astro. amu.edu.pl photometry and spectroscopy was confirmed in extensive photometric studies reported by Udalski \& Schwarzenberg-Czerny (1989; US89) and P97 and references therein. The latter authors advanced a plausible hypothesis, yet to be proven, relating this phenomenon to the superhump oscillations in SU UMa dwarf novae. Interestingly, P97 in their campaigns in 1992, 1993 and 1994 detected the superhump periods alternatively shorter $(0.1340 \mathrm{~d})$ and longer than the orbital one. They suggested that the effect was consistent with the hypothesis of the prograde motion of the line of apsides and the retrograde motion of the line of nodes in the accretion disk.

In June 1989, Schwarzenberg-Czerny, Udalski \& Monier (1992, hereafter SUM) obtained 28 IUE observations covering 5 orbital cycles and reported UV continuum pulsations with a period of $0.0437 \mathrm{~d}$. Motivated by a similar period pattern in TV Col, they suggested that this may be the rotation period of the white dwarf and thus V603 Aql might be an intermediate polar. US89 and P97 hinted at the possible presence of a weak related modulation in their photometry. However, in their detailed discussion of this issue, $\mathrm{P} 97$ note that given the negative results of Patterson et al. (1993) this may not be a permanent feature in V603 Aql. Hence, the intermediate polar hypothesis remained doubtful. The complexity of the periodic variations 
in V603 Aql and the dominance of the energy losses in the high energy bands in this system called for a detailed UV and $\mathrm{X}$-ray study. The high energy emission generally arises in several regions of a CV: its inner accretion disk with its boundary layer, its disk's wind and, occasionally, from a hot spot. In the present paper we report the results of our campaign of simultaneous multiwavelength observations of V603 Aql, the most extensive continuous UV observations so far for a cataclysmic variable.

Warner (1995) presented a comprehensive review of $\mathrm{X}$ and UV observations of CVs, including early HST observations. Summarizing, the average X-ray and emission line fluxes of CVs are mutually correlated with each other and with accretion rate (e.g. Patterson \& Raymond 1985). Less is known about the correlation of these properties in any individual CV as simultaneous X-UV observations are scarce. Strong UV emission lines from low inclination CVs show P-Cyg profiles, indicating strong wind outflow. Warner (1995) lists numerous evidence for the variability of P-Cyg profiles with orbital phase. This conclusion needs qualification as observations available then and now typically cover one or two orbital cycles. Hence, the evidence for variability is strong but a demonstration of coherent orbital periodicity is still missing. Typical HST observations are unsuitable for orbital modulation studies as they tend to be short and are not contiguous because of Earth occultation. The breakthroughs brought by HST observations are due to its superior temporal and spectral resolution. In particular, in several systems HST studies of eclipse modulation and line details enable determination of the size of the emission region and wind velocities.

Particularly relevant observations are discussed in Sect. 5 and apart from those quoted by Warner (1995, Sect. 2.7.3) involve papers by Szkody \& Mateo (1986), Friedjung et al. (1997), Ringwald \& Naylor (1998) and Prinja et al. (2000). Another preliminary report by Mauche et al. (2000) concerns the longer, albeit discontinuous, series of HST and multiwavelength observations of the magnetic system EX Hya. Our observations are highly complementary to those by HST in two respects. Namely, we adequately cover many (20) simultaneous orbital cycles, corresponding to a typical accretion time of the disc, enabling us to draw conclusions about orbital and (possible) accretion modulation. Our observations are discussed in Sect. 2. In Sect. 3 we investigate the presence of any periodic modulation in our data. Due to multiwavelength coverage and extended time coverage our observations yield a new capability to study the long term auto- and cross-correlation of multiwavelength signals (Sect. 4). Section 5 is devoted to a discussion of the variable P-Cyg profile of the CIV1550 line. In Sect. 6 we discuss the implication of our observations for existing accretion and wind models in CVs.

\section{Observations}

Our simultaneous observations in the UV and X-ray bands were obtained in October 1992. The X-ray observations obtained with the ROSAT satellite PSPC detector lasted $30 \mathrm{ks}$ and were distributed more-or-less evenly with a filling factor of 7 percent. Gaps in these observations arise due to

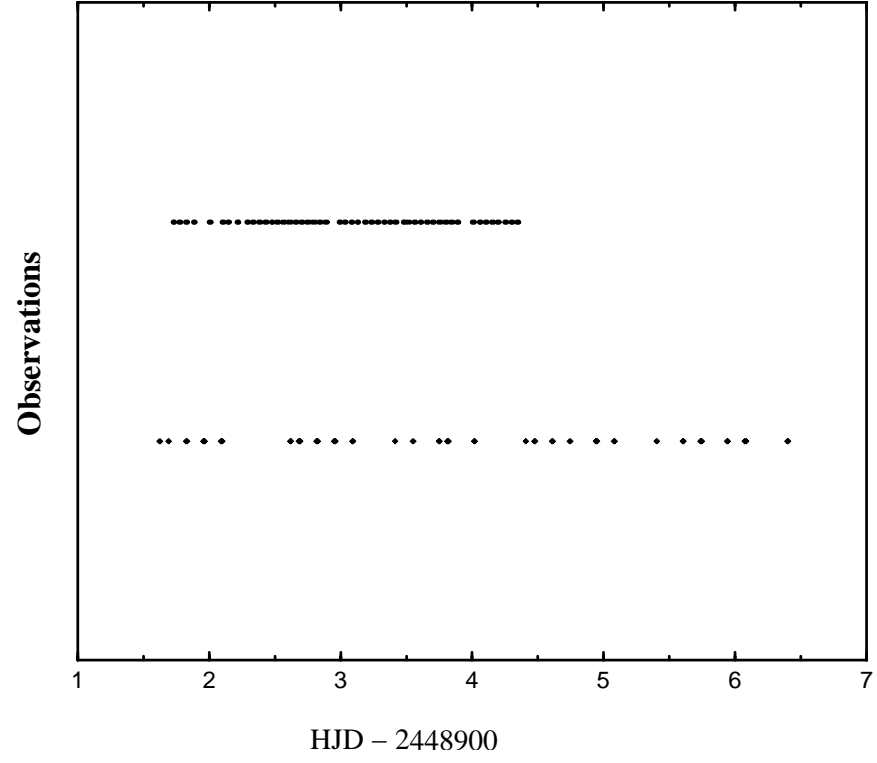

Fig. 1. Timing of IUE (top) and ROSAT (bottom) observations. Time is in JD minus 2448900 . Note that the orbital frequency of V603 Aql is $7.2 \mathrm{c} / \mathrm{d}$.

Earth occultation and satellite constraints on a low earth orbit. The UV observations were collected with the IUE satellite Short Wavelength Prime (SWP) camera in its low dispersion mode during a contiguous interval of 64 hs using 12 min exposures with a filling factor OF about 50 percent. Gaps lasting up to $25 \mathrm{~min}$ are caused by the read-prep operations of the satellite camera. Our time coverage with the ROSAT and IUE observations is shown in Fig. 1. The simultaneous X-ray and UV observations span almost 3 days, i.e. 20 binary orbits, while our total $\mathrm{X}$ ray observations span 40 orbits.

\subsection{IUE observations}

\subsubsection{Procedures}

The log of IUE observations is listed in Table 1. All SWP frames covered the wavelength band from 1000 to $1900 \AA$ and were well exposed.

As we expected the presence of a short-period modulation (due to US89), it was necessary to minimize the time between successive integrations. On the other hand, the satellite hardware restricts the time resolution to about one frame per $40 \mathrm{~min}$. To avoid this problem we decided to employ the technique invented by early spectroscopists - the "trailed spectra method".

This technique is based on double-exposed frames. The spectrograph slit size was large enough to let us expose 2 spectra on the same frame, parallel to each other. After the first exposure was done, we changed the attitude of the satellite and then the second exposure began. Then, the whole frame containing the two UV spectra ("A" and "B") was transmitted to the Earth. Thus, the time separation between adjacent spectra in one pair could be as short as needed, while the separation between succeeding frames was still restricted by the IUE hardware and READPREP operation. 
Table 1. IUE observation Log, $t=\mathrm{JD}-2448$ 900.0.

\begin{tabular}{crr|rrr}
\hline \hline SWP & & & SWP & & \\
number & $t_{A}$ & $t_{B}$ & number & $t_{A}$ & $t_{B}$ \\
\hline 45850 & 01.735 & 01.725 & 45851 & 01.772 & 01.782 \\
45852 & 01.832 & 01.820 & 45853 & 01.880 & 01.891 \\
45855 & 02.013 & 02.000 & 45856 & 02.051 & 02.065 \\
45857 & 02.097 & 02.109 & 45858 & 02.139 & 02.151 \\
45860 & 02.212 & 02.223 & 45862 & 02.286 & 02.299 \\
45863 & 02.329 & 02.340 & 45864 & 02.376 & 02.389 \\
45865 & 02.437 & 02.424 & 45866 & 02.473 & 02.483 \\
45867 & 02.528 & 02.511 & 45868 & 02.558 & 02.574 \\
45869 & 02.622 & 02.603 & 45870 & 02.652 & 02.667 \\
45871 & 02.712 & 02.697 & 45872 & 02.741 & 02.760 \\
45873 & 02.806 & 02.787 & 45874 & 02.835 & 02.848 \\
45875 & 02.897 & 02.880 & 45877 & 03.000 & 02.990 \\
45878 & 03.029 & 03.039 & 45879 & 03.079 & 03.089 \\
45880 & 03.125 & 03.135 & 45881 & 03.182 & 03.195 \\
45882 & 03.231 & 03.243 & 45883 & 03.277 & 03.290 \\
45884 & 03.327 & 03.337 & 45885 & 03.369 & 03.383 \\
45886 & 03.428 & 03.413 & 45887 & 03.476 & 03.486 \\
45888 & 03.524 & 03.505 & 45889 & 03.557 & 03.569 \\
45890 & 03.615 & 03.604 & 45891 & 03.652 & 03.665 \\
45892 & 03.710 & 03.699 & 45893 & 03.744 & 03.762 \\
45894 & 03.807 & 03.793 & 45895 & 03.836 & 03.854 \\
45896 & 03.897 & 03.885 & 45898 & 04.017 & 04.002 \\
45899 & 04.055 & 04.068 & 45900 & 04.101 & 04.113 \\
45901 & 04.147 & 04.163 & 45902 & 04.193 & 04.205 \\
45903 & 04.249 & 04.261 & 45904 & 04.295 & 04.307 \\
45905 & 04.340 & 04.352 & & & \\
\hline & & & & &
\end{tabular}

Because the spectrograph slit has finite height (in practice about $\left.20^{\prime \prime}\right)$, the spatial separation between two spectral images could not be as large as desired without flux losses. The result is that two spectra on the same frame overlap partially. This unwanted effect is increased by the defocusing: focusing of the IUE was very often switched off to maximize available power.

\subsubsection{Reductions}

Due to the partial overlap, it was impossible to separate the individual spectra with the standard IUESIPS software. Thus, we used IUESIPS only to perform a geometric and photometric correction to the raw image and used the resolved line-by-line spectra (LBLS) and MXLO/MELO files with calibrated fluxes for further calculations.

We started with the "old style" ELBL/MELO files produced by the older version of IUESIPS software. After the NEWSIPS software had been employed we repeated our procedure for new SILO/MXLO files. We obtained identical results for both sets of data.

In Fig. 2 we plot the section of a sample frame perpendicular to the dispersion. Our first attempt to deal with the overlapping spectra was based on fitting a double Gaussian function to the subsequent perpendicular sections of each spectra stored in the LBLS files. Fitting Gaussian functions gave us a possibility to estimate the values of the "overlapped" parts of adjacent spectra "A" and "B" and then calculate the real

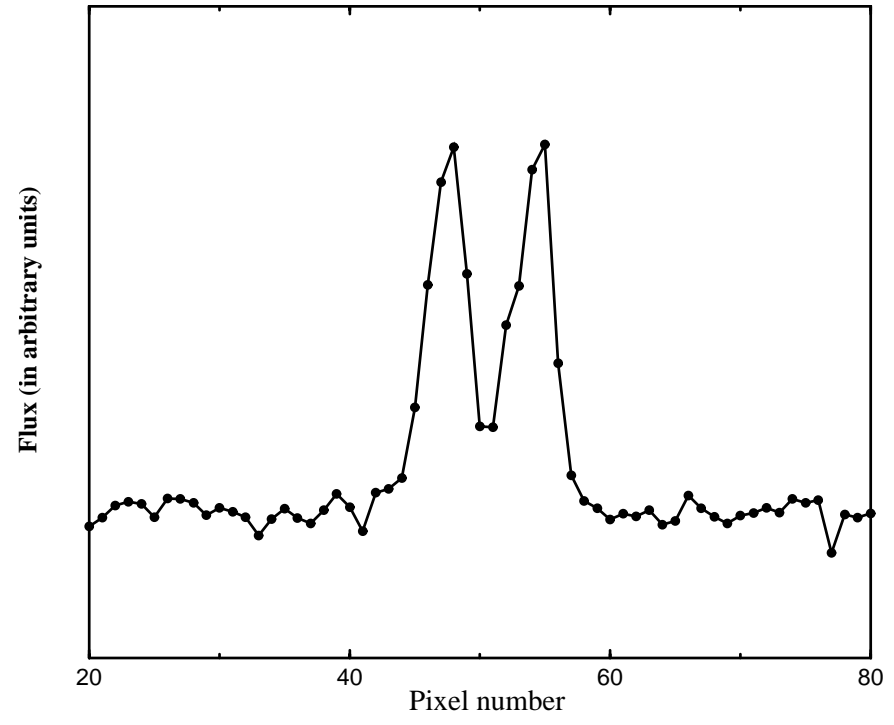

Fig. 2. Scan perpendicular to dispersion for a section of a raw spectrum (row $m=300$ ). Note the considerable overlap of individual exposures.

total contribution of both elements of the pair to the total flux. In this way we calculated quotients $\frac{A}{(A+B)}$ and $\frac{B}{(A+B)}$ for each row of pixels. After a simple multiplication of these quotients by the absolutely calibrated total flux values taken from the MELO/MXLO files for the same row, we obtained a pair of one dimensional sets of calibrated flux values with the corresponding wavelengths separately for " $\mathrm{A}$ " and "B" spectra.

Unfortunately, this algorithm did not work sufficiently well in many cases, particularly with the under exposed frames. It was also very sensitive to artifacts on the frames. It was clear that our rectification technique should be improved. We employed a novel method in order to extract individual spectra from the melted images, based on the Lucy-Richardson deconvolution method (Lucy 1974; Spruit 1994). This method helped us to reduce the spatial cross-talk of parallel spectra by numerical deconvolution. Details are described by Borczyk (1999).

The average properties of our extracted 1992 UV spectra, namely their average and standard deviation are displayed in Fig. 3. Note the excess variance in the blue wing of CIV1550 and the lack of it in the red wing. Comparison with the 1989 data reveals that in 1992, the scattering at continuum frequencies is largely due to flickering intrinsic to the star (cf. Sect. 2.1.4). Note also that the relative scatter of the continuum is wavelength independent.

\subsubsection{Light curves and colors}

Photometric UV measurements were obtained by integrating our individual calibrated UV spectra over selected wavelength bands. Five bands were selected to cover broad continuum spectral regions that were little affected by lines: 1275-1375, $1425-1475,1575-1625,1650-1750$ and 1800-1900 ̊. To be able to investigate changes in the continuum gradient we used UV color defined as

$C=-2.5 \log _{10}\left(F_{1275-1375} / F_{1800-1900}\right)$. 


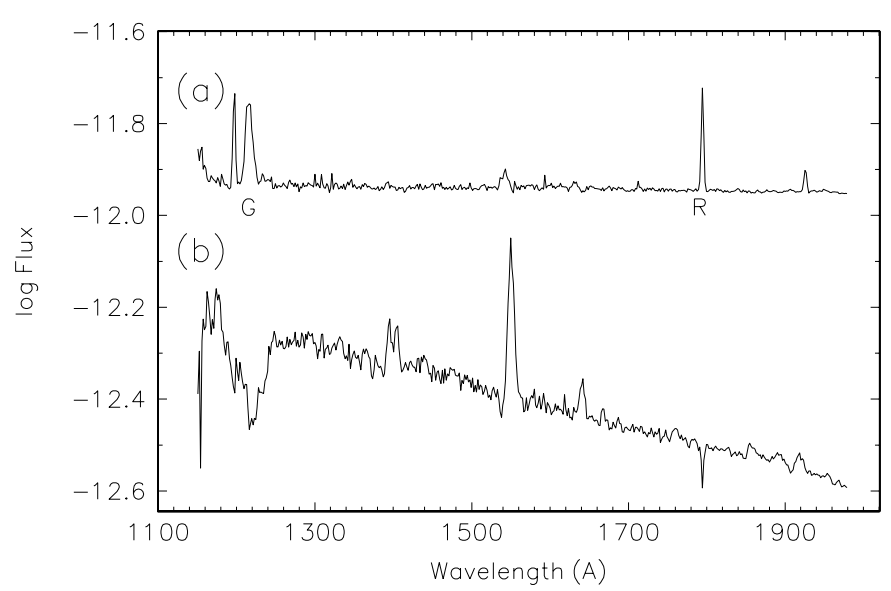

Fig. 3. Statistical properties of UV spectra: standard deviation per single spectrum (a, shifted by -12) and average spectrum (b), both in log Flux units, where flux is in (erg cm $\left.\mathrm{cm}^{-2} \mathrm{~s}^{-1} \AA^{-1}\right)$. Letters $(\mathrm{G})$ and (R) mark structures affected respectively by the geocoronal radiation and reseau mark.

Additionally we selected narrow bands covering the emission components of the strongest lines of carbon CIV1550 and the silicon SiIV1393/1402 A doublet and the blue-shifted variable absorption component of CIV1550. For this purpose we linearly interpolated the continuum on both sides of a line and subtracted it from the flux within the line. The lightcurves for the continuum (1575-1625 band) and the CIV1550 line are shown in Fig. 4. We already remarked on the scatter of the UV fluxes in Sect. 2.1.2. The distribution of the continuum UV values is discussed below, in Sect. 2.1.4. For the lines, see the discussion of Figs. 14 and 18. The mean value and standard deviation of the color $C$ (Eq. (1)) was, respectively, -0.575 and $0.071 \mathrm{mag}$. No trend was seen in the color vs. time plot.

\subsubsection{Verification and comparison with 1989 data}

To verify our new IUE reduction procedure for the multiple exposed spectra, we processed, in the same way as the present data, the archival IUE observations of V603 Aql and V795 Her from 1989 and 1992, and extracted their line and continuum lightcurves. Since the recovered light curves were consistent with the behavior described in the original publications by Schwarzenberg-Czerny et al. (1992) and Prinja \& Rosen (1993) based on independent reductions, we conclude that our reduction procedure is correct.

Specifically, our newly reduced old 1989 data for V603 Aql did reveal the pulsations reported by SUM and did not exhibit coherent modulation in the lines (cf. Schwarzenberg-Czerny et al. 1992). We shall demonstrate that our present observations exhibit quite contrary results on both accounts. In this respect it must be stressed that our new 1992 data are more reliable as they contain 5 times more observations than the 1989 ones. However, it may be premature to solely blame poor statistics for the discrepant 1989 observations. In Fig. 5 we present the 1989 continuum light curve (1575-1625 $\AA$ band). Despite the identical instrument setup, the observing procedures and

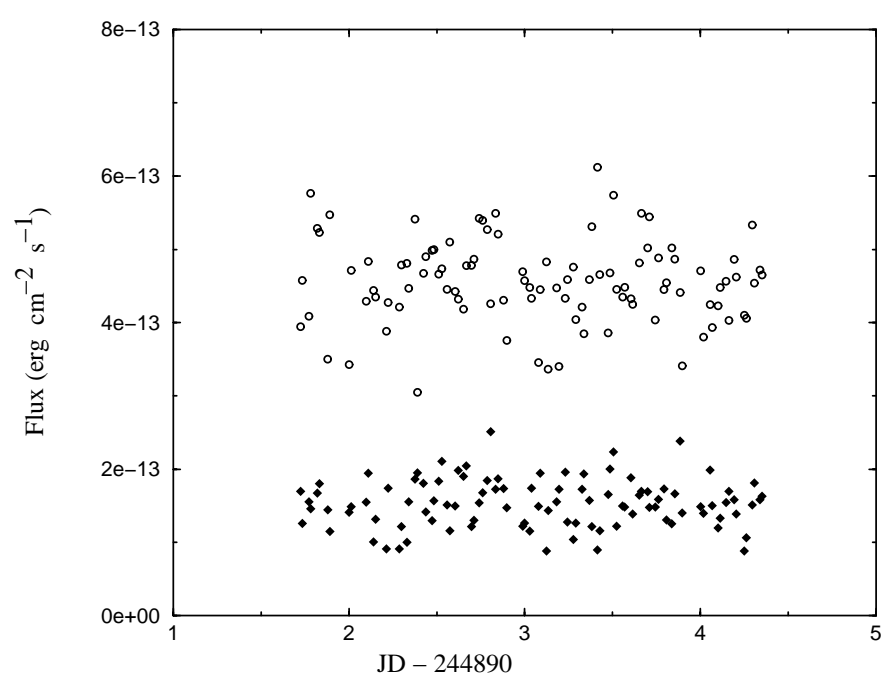

Fig. 4. Continuum (wavelength band 1575-1625 ̊) and CIV1550 emission line lightcurves. The continuum points are denoted by black diamonds and the CIV1550 points by open circles. The time is in JD minus 2448900 .

the data reduction techniques, the amplitude of the scatter of the 1989 continuum and line observations amounts to only a third of the corresponding values in 1992, of 40 and 30 percent. Hence it is difficult to avoid a conclusion that V603 Aql was in different states in 1989 and 1992, at least in some respects. It remains an open question whether these differences in states affected the presence and/or disappearance of any intrinsic oscillations.

Photometry of V603 Aql was obtained by Francois Piche with the $0.76 \mathrm{~m}$ telescope of the University of Washington (WA) during the 1992 satellite observations. According to the results kindly communicated to us they reveal a range in brightness between 11.65-12.0 mag. The AAVSO data from June 1989 indicate a similar range, between 11.311.8. The decline of the average brightness by $0.2 \mathrm{mag}$ in $3 \mathrm{yrs}$, if real, would be consistent with the average rate of the decline 70 yrs after outburst.

\subsection{ROSAT observations}

The X-ray observations were obtained by the ROSAT satellite in the pointing mode with the PSPC(2) detector over $30 \mathrm{ks}$. The satellite and its instrumentation are described by Trumper (1983). For further details see Voges (1992). Due to satellite constraints and Earth occultation, the observations of V603 Aql occur in 19 intervals lasting no more than 25 min each. The observations were reduced in a standard way using the EXSAS interactive software package (Zimmermann et al. 1992). Analysis of the spectral properties of V603 Aql will be published elsewhere. By selecting and binning photons into standard PSPC/SASS energy bands we obtained lightcurves in $A, B, C, D$ energy bands plus an integrated light curve corresponding to $0.3-2 \mathrm{keV}$. Variability in each of the energy bands had a similar character. We discuss here in detail only the integrated PSPC light curve because its better statistics yielded tighter upper limits on any correlations and periodic 


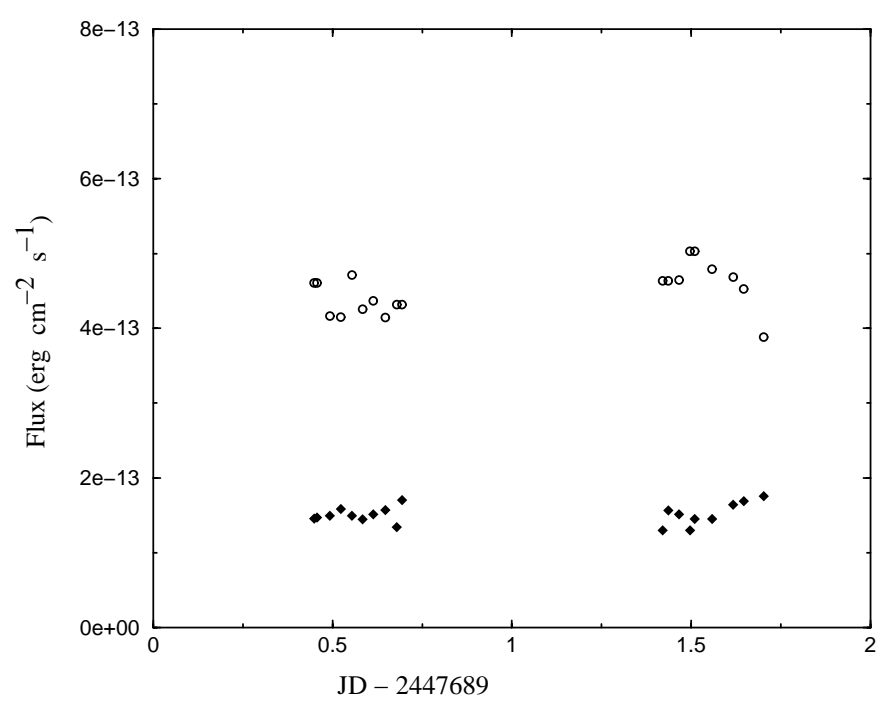

Fig. 5. Same as Fig. 4 for observations from 1989 except that the horizontal axis id JD minus 2447689 . Note that the scatter of observations is one third that observed in 1992 with the same instrument setup and reduction techniques.

modulation. As a compromise between the time resolution and sufficient count rate per bin, we binned our lightcurves into $400 \mathrm{~s}$ time bins.

If we denote the flux value of point $(x, y)$ from LBLS file by $\Phi(x, y)$, the rth approximation of the real (deconvolved) flux value in point $(\xi, \eta)$ by $\Psi^{r}(\xi, \eta)$ and the convolution kernel by $P(x-\xi, y-\eta)$ then the iterative scheme can be described as:

$\Psi^{r+1}(\xi, \eta)=\Psi^{r}(\xi, \eta) \iint \frac{\Phi(x, y)}{\Phi^{r}(x, y)} P(\xi-x, \eta-y) \mathrm{d} x \mathrm{~d} y$

where

$\Phi^{r}(x, y)=\iint \Psi^{r}(\xi, \eta) P(x-\xi, y-\eta) \mathrm{d} \xi \mathrm{d} \eta$.

Substituting a Gaussian function as the convolution kernel and assuming that we can approximate parameters of this function we can start our iterative process from $\Psi^{0}=\Phi$

In most cases the iterative process converged rapidly to the final solution and enabled us to avoid fitting Gaussian functions to the sections of spectra: after this procedure adjacent spectra were sufficiently well separated and no additional rectification was needed - the "contribution quotients" of "A" and "B" were then easy calculated by a simple summation of the flux values in the proper areas of the deconvolved image.

Lucy's algorithm proved to be an efficient and powerful tool to process our data. All UV observations referred below were reduced in this way. We processed all 50 frames containing double exposed spectra and obtained 100 single spectra as a result. The log of the observations is listed below. The first column contains the frame number, the second and the third are the mid-times of exposure for the "A" and "B" spectra (Julian day number minus the constant offset $=2448900.0$ ) The barycentric corrections had been included before the observations were used to calculate the light curve.
Table 2. ROSAT observations.

\begin{tabular}{cccc}
\hline \hline Start date & Start time UT & End date & End time UT \\
\hline $6-10-92$ & $02: 51: 35$ & $6-10-92$ & $03: 14: 20$ \\
$6-10-92$ & $04: 15: 58$ & $6-10-92$ & $04: 51: 54$ \\
$6-10-92$ & $07: 43: 53$ & $6-10-92$ & $08: 06: 17$ \\
$6-10-92$ & $10: 39: 47$ & $6-10-92$ & $11: 19: 39$ \\
$6-10-92$ & $13: 56: 15$ & $6-10-92$ & $14: 35: 02$ \\
$7-10-92$ & $02: 35: 57$ & $7-10-92$ & $03: 08: 58$ \\
$7-10-92$ & $04: 13: 57$ & $7-10-92$ & $04: 46: 13$ \\
$7-10-92$ & $07: 22: 43$ & $7-10-92$ & $08: 00: 13$ \\
$7-10-92$ & $10: 34: 22$ & $7-10-92$ & $11: 13: 28$ \\
$7-10-92$ & $13: 49: 36$ & $7-10-92$ & $14: 29: 28$ \\
$7-10-92$ & $21: 43: 25$ & $7-10-92$ & $22: 08: 39$ \\
$8-10-92$ & $00: 54: 43$ & $8-10-92$ & $01: 26: 13$ \\
$8-10-92$ & $05: 45: 10$ & $8-10-92$ & $06: 17: 29$ \\
$8-10-92$ & $07: 17: 53$ & $8-10-92$ & $07: 55: 00$ \\
$8-10-92$ & $12: 06: 56$ & $8-10-92$ & $12: 44: 31$ \\
$8-10-92$ & $21: 40: 45$ & $8-10-92$ & $22: 03: 23$ \\
$8-10-92$ & $23: 14: 05$ & $8-10-92$ & $23: 43: 23$ \\
$9-10-92$ & $02: 26: 25$ & $9-10-92$ & $02: 57: 43$ \\
$9-10-92$ & $05: 37: 08$ & $9-10-92$ & $06: 12: 13$ \\
$9-10-92$ & $10: 25: 06$ & $9-10-92$ & $11: 01: 43$ \\
$9-10-92$ & $13: 39: 14$ & $9-10-92$ & $14: 19: 14$ \\
$9-10-92$ & $21: 33: 41$ & $9-10-92$ & $21: 58: 23$ \\
$10-10-92$ & $02: 20: 52$ & $10-10-92$ & $02: 52: 28$ \\
$10-10-92$ & $05: 32: 48$ & $10-10-92$ & $06: 06: 27$ \\
$10-10-92$ & $10: 19: 25$ & $10-10-92$ & $10: 55: 57$ \\
$10-10-92$ & $13: 36: 29$ & $10-10-92$ & $14: 14: 42$ \\
$10-10-92$ & $21: 29: 12$ & $10-10-92$ & $21: 53: 09$ \\
\hline & & &
\end{tabular}

\section{Fourier analysis of UV and $X$ lightcurves}

Further discussion in the present section and in Sect. 4 is based on the following time series: UV continuum, $F_{1575-1625}$ (Sect. 2.1.3), UV color $C$ (Eq. (1)), combined PSPC X-ray count rate $R_{X}$ (Sect. 2.2) and flux in the emission line CIV1550 ^ (Sect. 2.1.3). Some trials with other energy band combinations did not yield results much different from these presented here. However, the number of different combinations soon became unwieldy.

For period analysis we used both power spectra and AoV periodograms (Schwarzenberg-Czerny 1989). No significant features were found in the [take out either] periodograms for UV continuum, UV color and X-ray cont rate. The periodogram for the CIV emission revealed the presence of an oscillation. The last two periodograms are discussed below in some detail.

\subsection{No evidence for an intermediate polar}

Periodograms were calculated for both the UV and X-ray observations to search for any oscillations. We covered a range from 0 to 70 cycles per day. The periodogram for the entire $\mathrm{X}$-ray band observed by the PSPC detector is displayed in 
Fig. 6. There is no evidence of any periodic modulation of the X-ray radiation. Similar but noisier results are obtained separately for the soft and hard PSPC bands. No modulations are seen in the 1992 UV continuum observations. This contradicts our claim of a $23 \mathrm{c} / \mathrm{d}$ modulation of the UV continuum observed in 1989 (Schwarzenberg-Czerny et al. 1992, SUM). Clearly the $23 \mathrm{c} / \mathrm{d}$ oscillation may not be persistent. Note that the 1992 UV observation lasted 5 times longer than the 1989 one. Intermediate polars generally exhibit persistent $\mathrm{X}$-ray pulsations. In view of the absence of X-ray pulsations in the V603 Aql X-ray lightcurve and the lack of persistent UV and optical modulation, our current data do not support the proposed classification of V603 Aql as an intermediate polar.

To check our new reduction procedures we re-extracted lightcurves from the 1989 raw spectra images and produced light curves in the same way as for the 1992 data. We recovered the lightcurves and UV continuum periodogram with the $23 \mathrm{c} / \mathrm{d}$ feature resembling those obtained by SUM, demonstrating that our new and old UV reductions are mutually consistent. Since our instrumental setup and reductions for 1989 and 1992 were similar, the $23 \mathrm{c} / \mathrm{d}$ feature might be both real and transient. Some evidence of a change intrinsic to the star comes from a factor of 2 increase in UV flickering between 1989 and 1992. While our 1992 optical lightcurves are too short to adequately search for this feature, possible optical detection of a related $23 \mathrm{c} / \mathrm{d}$ quasi-periodic oscillation is discussed by Patterson et al. (1997).

\subsection{Periodic modulation of the lines}

In strong contrast to the UV continuum, the periodogram of the emission line intensity indicates modulation with a frequency of $7.24 \mathrm{c} / \mathrm{d}$ in both the CIV1550 and SiIV1393/1402 $\AA$ doublet lines. This frequency agrees with the orbital period previously found from the radial velocities of the optical lines. The distinct peak near the frequency $v_{1} \approx 7.24 \mathrm{c} / \mathrm{d}$ is easily visible in Fig. 7 . There is no doubt that it corresponds to a real effect.

Less certain is the reality of a high frequency feature around $v_{2} \approx 42.71(6) \mathrm{c} / \mathrm{d}$, which has never been seen before and has no simple relation to any frequencies claimed in the past. The high frequency feature is rather robust and persists after prewhitening of the other (orbital) frequency. This should be no surprise as our window function is particularly clean and devoid of any artifacts exceeding the 10 percent level. Since our 1992 data are quite extensive, the periodogram feature is quite clean and its frequency does not exceed half of the maximum sampling frequency of order $100 \mathrm{c} / \mathrm{d}$, we must conclude that the $v_{2}$ modulation is real and at least temporarily persistent. As the width of the $v_{1}$ and $v_{2}$ features are similar, we have no evidence of any incoherence of the latter modulation. It is rather frustrating to observe that $v_{2} / 2$ is close but significantly different from any possible frequencies discussed in V603 Aql in the past (US89, SUM, P97). However, until more evidence is available, we refrain from further discussion of the nature of any related process. Our effective Nyquist frequency is about $50 \mathrm{c} / \mathrm{d}$, so the feature at $64.8 \mathrm{c} / \mathrm{d}$ could be a ghost of a peak at lower frequency.

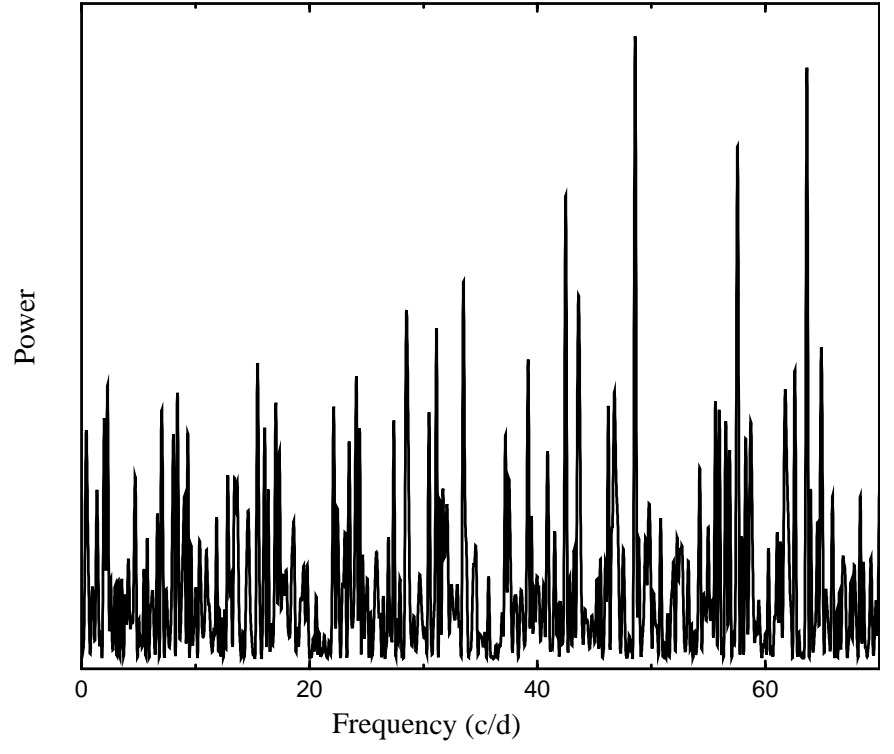

Fig. 6. Power spectrum for the integrated X-ray lightcurve in $400 \mathrm{~s}$ bins. On the horizontal axis is frequency in cycles per day.

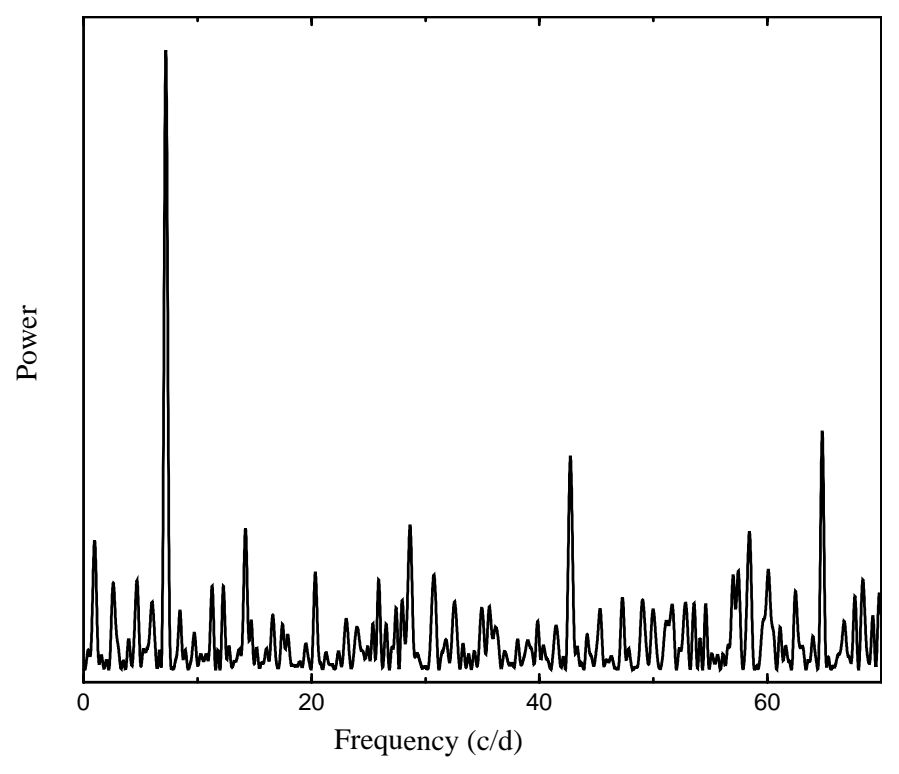

Fig. 7. Power spectrum for the CIV $1550 \AA$ A emission line.

\section{Cross-correlation and auto-correlation analysis}

\subsection{Analysis for large time lags}

To study the cross-correlation between the data series listed at the beginning of Sect. 3 we adopted the method by Edelson \& Krolik (1988). For each pair of two data series $a_{i}(t)$ and $b_{j}(t)$ we calculate its time lag $\Delta t_{i j}=t_{j}-t_{i}$ and its contribution to the unbinned discrete cross-correlation function

$U C C F_{i j}=\frac{\left(a_{i}-\bar{a}\right)\left(b_{j}-\bar{b}\right)}{\sqrt{\left(\sigma_{a}^{2}-e_{a}^{2}\right)\left(\sigma_{b}^{2}-e_{b}^{2}\right)}}$

where $e_{a}$ and $e_{b}$ are the measurement errors associated with the corresponding data sets. In our case we set both parameters 
equal to zero. Eventually the discrete cross-correlation function $C C F(\tau)$ is calculated by binning and averaging the UCCF in time lag. A similar procedure applies to the calculation of the auto-correlation function (ACF) of each individual time series.

We investigated ACF for the following time series: log UV continuum flux, UV color, log X-ray flux and CIV1550 emission. Most of them revealed nothing except for the feature at 0 lag, to be expected by default (see examples in Figs. 8 and 9). The real effect in the ACF was found for CIV1550 emission. This is predictable, as the correlation of a periodic function with an arbitrary function is also a periodic function. This is best seen by noting that the periodic function shifted by its period is identical with itself. Since we found the orbital modulation of the CIV1550 line (Sect. 3.2), the orbital modulation with the period of $0.138 \mathrm{~d}$ reappeared in any correlation involving CIV1550. This effect for the ACF CIV1550 is shown in Fig. 10.

There are $\left(\begin{array}{l}4 \\ 2\end{array}\right)=6$ different combinations of our data for the CCF. Among them, the 3 which involve the CIV1550 line display periodic features as discussed in the preceding paragraph (e.g. Fig. 11). Nothing significant was observed in X-rays vs. UV color nor in UV continuum vs. UV color. In the latter case, the slight anti-correlation at 0 lag is not significant at $2.3 \sigma$ level. The X-ray vs. UV continuum plot revealed a feature at 0 lag and nothing for lags exceeding $10 \mathrm{~min}$. Note that for CCF no such feature is to be expected by default hence we discuss this case in Sect. 4.2. The cross-correlation function for UV color vs. X-ray flux is presented in Fig. 12 and discussed in Sect. 4.3.

\subsection{Correlation of UV and X-ray fluxes}

We calculated the CCF for UV and X-ray fluxes for time lags extending from $-2 \mathrm{~d}$ till $+2 \mathrm{~d}$. For lags $\leq-1 \mathrm{~d}$ our plot becomes noisy because of dearth of the X-ray data sampled before the UV observations. The only structure visible in the remaining part of the plot is located at 0 lag. In order to investigate it further we took a small interval time $\Delta t$ centered at 0 and selected all $n$ pairs of X-ray and UV continuum observations separated by no more than $\Delta t$. Next we calculated their correlation coefficient $\rho$ and the statistics

$t=\frac{\rho}{\sqrt{1-\rho^{2}}} \sqrt{n-2}$.

A shorter $\Delta t$ for higher $\rho$ was observed except for the smaller sample of $n$ observations: for $\Delta t=0.0398 \mathrm{~d} \rho=0.273$ and $n=115$, while for $\Delta t=0.00316 \rho=0.768$ and $n=13$. For the null hypothesis $H_{0}$ assuming no correlation, i.e. $\rho=0$, the $t$ statistics obeys the Student distribution with $n-2$ degrees of freedom (Fisz 1963). For the majority of values of $\Delta t$ in the range given above, we obtained values of $t$ consistent with rejection of $H_{0}$ at $3 \sigma$ level (two-sided test). However, the correlation was never more significant than $3.9 \sigma$ for $\Delta t=0.00501$ and $\rho=0.773$ (cf. Fig. 13). Neither the observed progression of increasing $\rho$ with decreasing $\Delta t$ nor the observed significance levels prove the existence of the correlation, but taken together they increase the reality of the observed correlation.

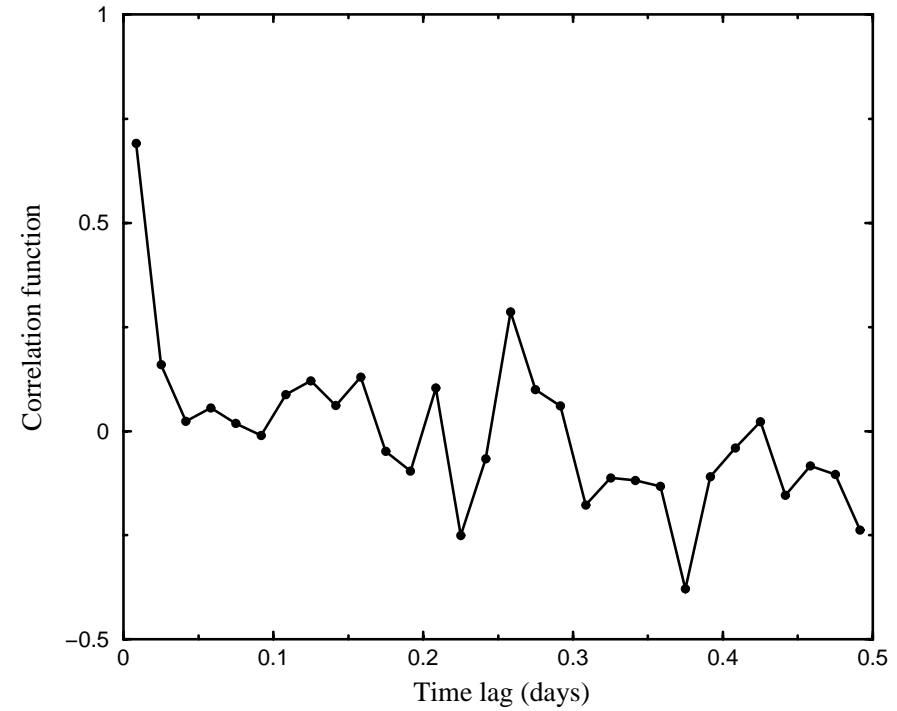

Fig. 8. Autocorrelation function for UV continuum. On the horizontal axis is the time lag in days.

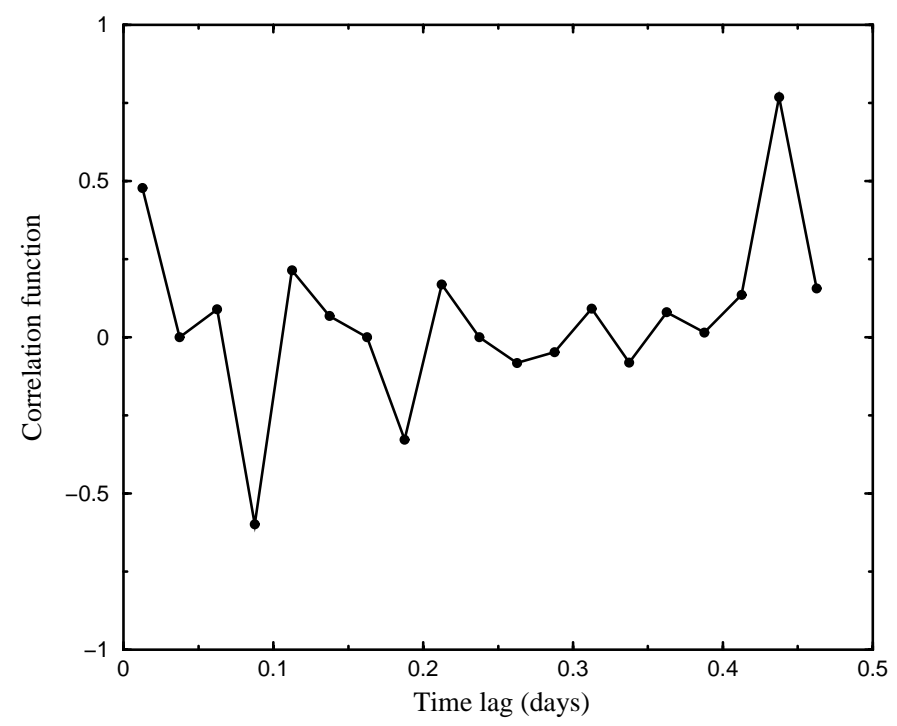

Fig. 9. Autocorrelation function for X-ray. Scales as in Fig. 8.

\subsection{Do $X$-rays trail modulation of the UV color?}

Figure 12 reveals the presence of conspicuous structure around the lag of $+0.11 \mathrm{~d}$. This lag roughly corresponds to the orbital period, hence it deserves some attention. Unlike most structures due to noise it extends over a whole interval of lags and it remains there for a range of time lag grids selected for calculation of the CCF following Edelson \& Krolik (1988). To verify our calculations we repeated the calculations using a different method. Namely, for each time-lag interval $l$ we selected all pairs of points from both data sets with time lags falling into the interval. Then we calculated the correlation coefficient $\rho$ of the values in the selected pairs. Our plot of $\rho(l)$ resembles that in Fig. 12. Thus we are confident that the feature at $+0.11 \mathrm{~d}$ is not a numerical artefact. A correlation analysis similar to that performed in Sect. 4.2 yields for lags $+0.11 \pm 0.005 \rho=0.585$, $n=17$ and the significance level $3.4 \sigma$. In summary, there is 


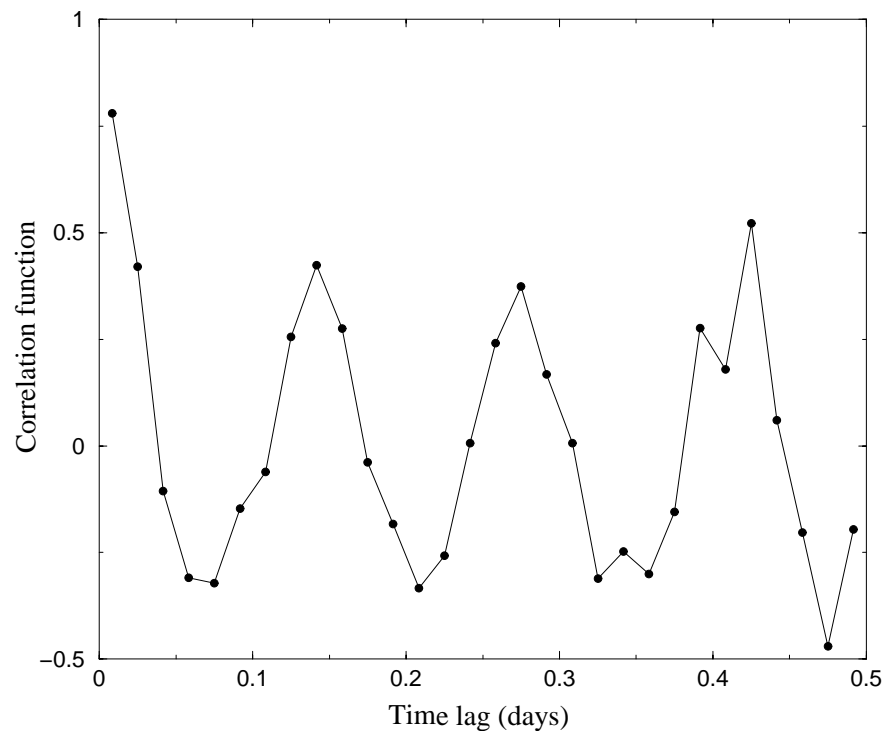

Fig. 10. Autocorrelation function for CIV1550 ̊ emission line (vertical axis). Scales as in Fig. 8. Note that the maxima repeat with the orbital period.

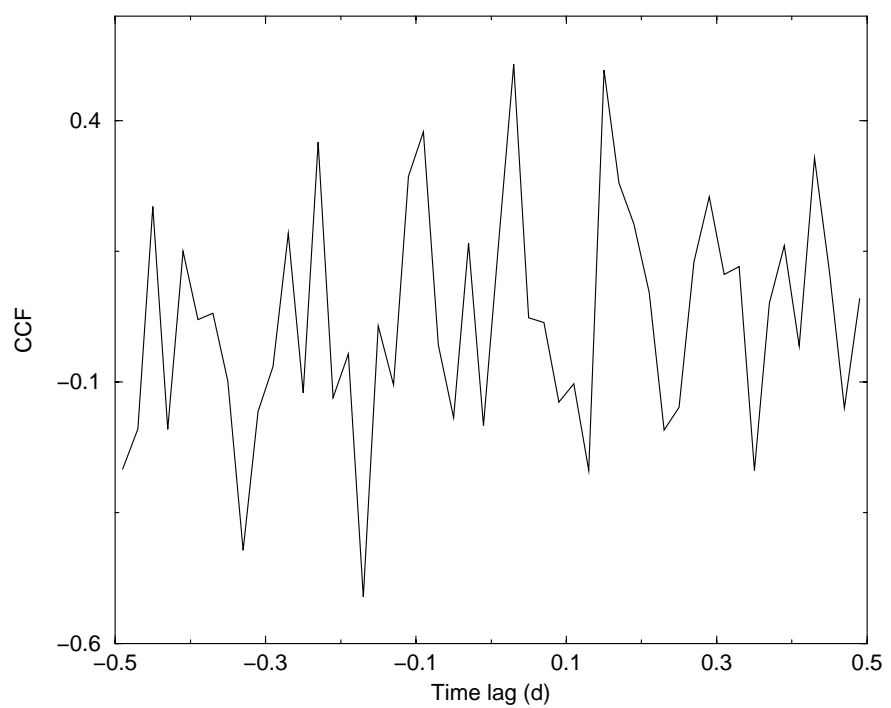

Fig. 11. Cross-correlation function (CCF) for CIV1550 emission vs. $\mathrm{X}$-ray integrated flux (vertical axis). Horizontal axis is time lag in days. The lag is positive when X-ray variations trail behind CIV1550. Note a periodic structure related to orbital modulation of CIV1550 (see text).

some indication of the cross-correlation of X-ray flux with the UV color, such that the X-ray variations are delayed with respect to the UV color by about an orbital period. The time scale and the cause of the effect might be related to an orbital modulation and/or accretion time scale of the inner region of the disc. However, the evidence proving the reality of this correlation is not overwhelming, hence we refrain from further speculations on its nature.

\section{P-Cygni profiles}

Szkody et al. (1986) undertook a pilot study of the modulation of P-Cyg profiles in the spectra of dwarf novae, specifically

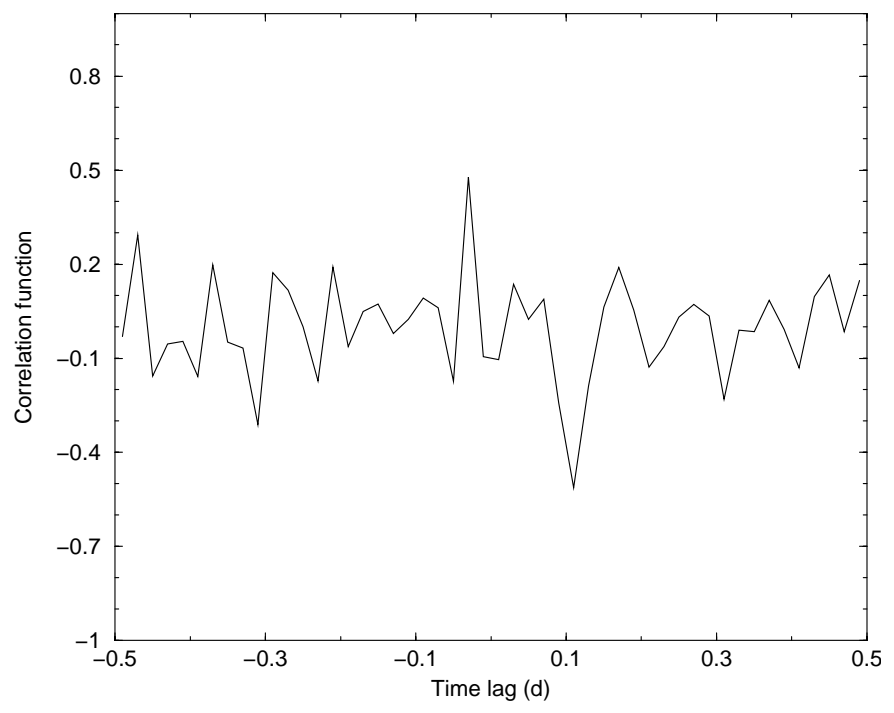

Fig. 12. The same as in Fig. 11 for UV color vs. X-ray integrated flux. Note the feature at the lag $+0.11 \mathrm{~d}$ discussed in Sect. 4.3.

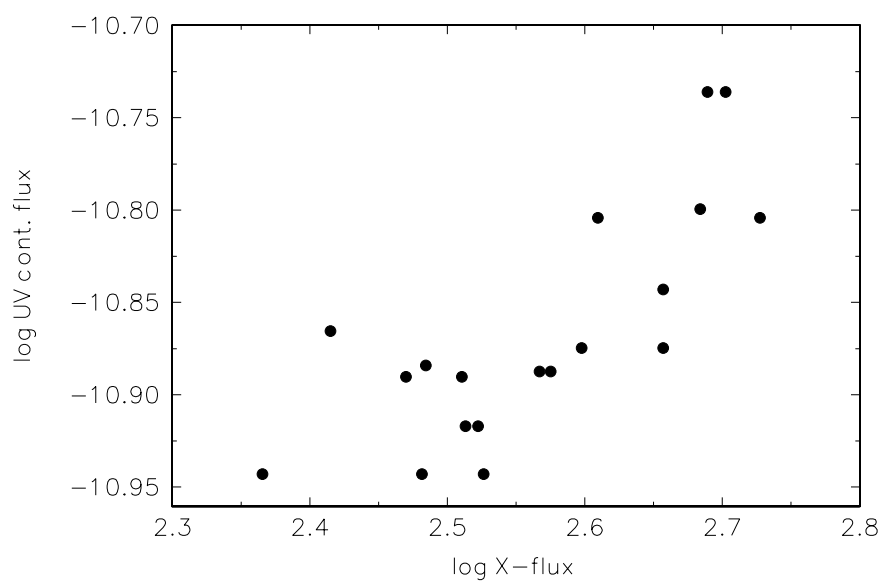

Fig. 13. UV continuum against $X$-ray flux at zero lag $(<0.00501 \mathrm{~d}) . X$-units: PSPC counts per $400 \mathrm{~s}$ time bin, $y$-units: erg $\mathrm{cm}^{-2} \mathrm{~s}^{-1} \AA^{-1} / 30$. Note that clustering around the diagonal indicates a positive correlation. According to Student $t$-test (Fisz 1963) the correlation is significant at the level $P=0.0001$, corresponding to $3.9 \sigma$.

in the CIV1550, SiIV1393/1402 doublet and NV1240 $\AA$ lines in Z Cam. Their observations were obtained over 2 IUE shifts on 10 and 12 December 1983, covering about 2 orbital cycles of Z Cam and, similarly to our data, using the large-aperture and low resolution mode. Szkody et al. (1986) reported that in both cycles the maximum of the absorption corresponds to the phases $0.6-0.8$ and suggested a possible role of the mass-transfer stream. However, dearth of data prevented a periodogram analysis and firm establishment of the coherent nature of the discovered modulation. Additionally these authors noted that the strength of the P-Cygni absorption is greater after outburst than during the standstill, while the final velocities and wavelengths of the central absorption component remain the same. No corresponding changes in the emission component of the CIV1550 lines were observed. 
Friedjung et al. (1997) used HST high dispersion ultraviolet spectra to discover the P-Cygni-like profiles of the CIV1550 line of V603 Aql. They also noticed that despite apparent similarity to "typical" P-Cygni profiles, this structure in V603 Aql exhibits a number of peculiarities. For example, the emission part is much stronger then the absorption one and almost exactly symmetrical. As a possible explanation, he suggests that the source of the emission lines is the hot, external part of the chromosphere of the disk, while the absorption lines are created in the colder stellar wind.

Friedjung et al. (1997) also noticed that the intensity of the lines varies on a time scale of minutes. Further detailed analysis of this effect was hampered since their observations covered only a fraction of the orbital period. Similar results were reported by Selvelli et al. (1998). Using 108 IUE SWP observations, part of which belongs to the present data, they were unable to find definite periodicities in the variations in the absorption features. They reported that the CIV1550 and NV1240 A lines reveal only irregular variations, indicative of variable wind outflow and of sudden ejection of optically thick material with time-scales of the order of $10 \mathrm{~min}$. Note however, that these authors performed only a preliminary study of the observations, did not reduce all double exposed frames and attempted no state-of-art periodogram analysis.

Our quest for high temporal resolution forced us to use the low resolution mode of IUE. This mode is considered to yield an insufficient resolution for studies of spectral line profiles. Still, given the large size of our dataset, we decided to take a closer look at the P-Cygni profiles. Our attempt was vindicated when it turned out that the width and shapes of the emission and absorption components in our data sometimes closely matched those obtained with HST GHRS. Coordination with ROSAT and long time coverage, rather difficult to attain with HST, were a bonus in our study. However, HST complements our results in providing high temporal resolution.

At first, we prepared the average spectrum from 100 IUE spectra. The vicinity of the CIV1550 line in this spectrum is shown in Fig. 14.

The absorption depression is easily visible on the short wavelength side of the CIV1550 A emission line.

Figure 15 shows the vicinity of the CIV 1550 line at different phases $\phi$ of orbital motion ( $\phi$ is relative only).

Notice that the absorption part of the profile changes in time. Furthermore, measuring the position of the absorption maximum and of the blue edge, we can determine the average and highest values of the radial velocity of the gas as $2100 \mathrm{~km} \mathrm{~s}^{-1}$ for the maximum absorption and $3100 \mathrm{~km} \mathrm{~s}^{-1}$ for the blue edge. Both values are measured in relation to the maximum of the emission. The radial velocity corresponding to the maximum of emission, measured by Friedjung et al. (1997) is equal to about $50 \mathrm{~km} \mathrm{~s}^{-1}$ and can be neglected in the present circumstances.

Radial velocities obtained from our IUE observations are similar to values calculated by Friedjung (1400 to $2100 \mathrm{~km} \mathrm{~s}^{-1}$ for the maximum of absorption and $2500 \mathrm{~km} \mathrm{~s}^{-1}$ for the blue edge). This suggests that the P-Cyg profile in our IUE low resolution data is sufficiently resolved.

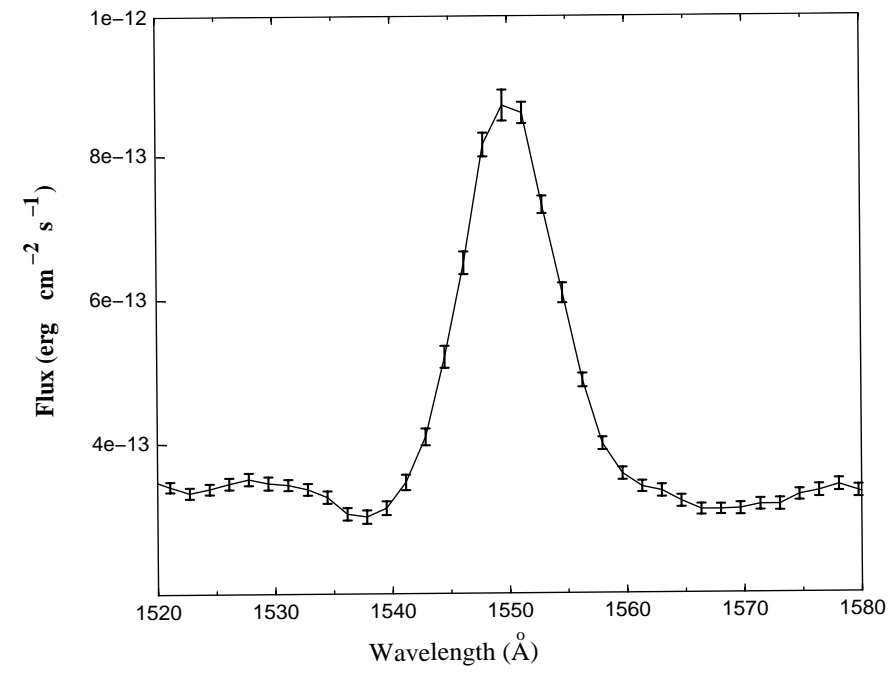

Fig. 14. Average profile of CIV1550 Å line, from 100 IUE spectra. On horizontal axis wavelength in $\AA$. Error bars indicate $\pm \sigma$ range of the mean. The scatter of the individual spectra is 10 times more.

We proceed to perform the analysis of the long term variability of the absorption and the emission components of the CIV1550 ̊ line (Sect. 2.1.3). The analysis of the absorption component lead to the discovery of the modulation with the period close to the orbital one. This is clearly visible in the periodogram for the absorption component in Fig. 16. Note the presence of a strong first harmonic of the orbital frequency. The power in the harmonic compared to that for the base frequency is much greater than its strength for the emission component (Fig. 7). Since the sampling of the lightcurves for the emission and absorption components is the same, the difference in the relative power reflects a true difference in the absorption and emission lightcurves and not any sampling/window function effects.

We obtained final estimates of the frequencies by fitting the lightcurves with a Fourier series truncated at the second harmonic. The residuals from the fit are uncorrelated, hence our error estimates should be realistic (Schwarzenberg-Czerny 1991, 1996). The results for the emission and absorption components of the CIV1550 line are compared in Table 3. Additionally in the table we list orbital and photometric periods obtained by Patterson et al. (1997) from independent observations made between 3-8 June 1992, 3-26 June 1992, 24 July-11 August and 8 August-14 September. We intentionally used results of photometric observations obtained at almost the same time as our IUE observations. These results suggest that the frequencies of modulation of the emission and absorption components of CIV1550 line are the same within $1.3 \sigma$ and equal to the orbital frequency. Indeed the weighted mean of the two former frequencies and the latter frequency are in excellent agreement. However, the average frequency of the CIV1550 modulation differs from the average photometric period by as much as $6 \sigma$. So it seems safe to conclude that the CIV1550 line is modulated with the orbital period and not with any of the photometric ones.

In Fig. 17 we display the phase folded and binned light curve of the CIV1550 emission line. Note the large amplitude 


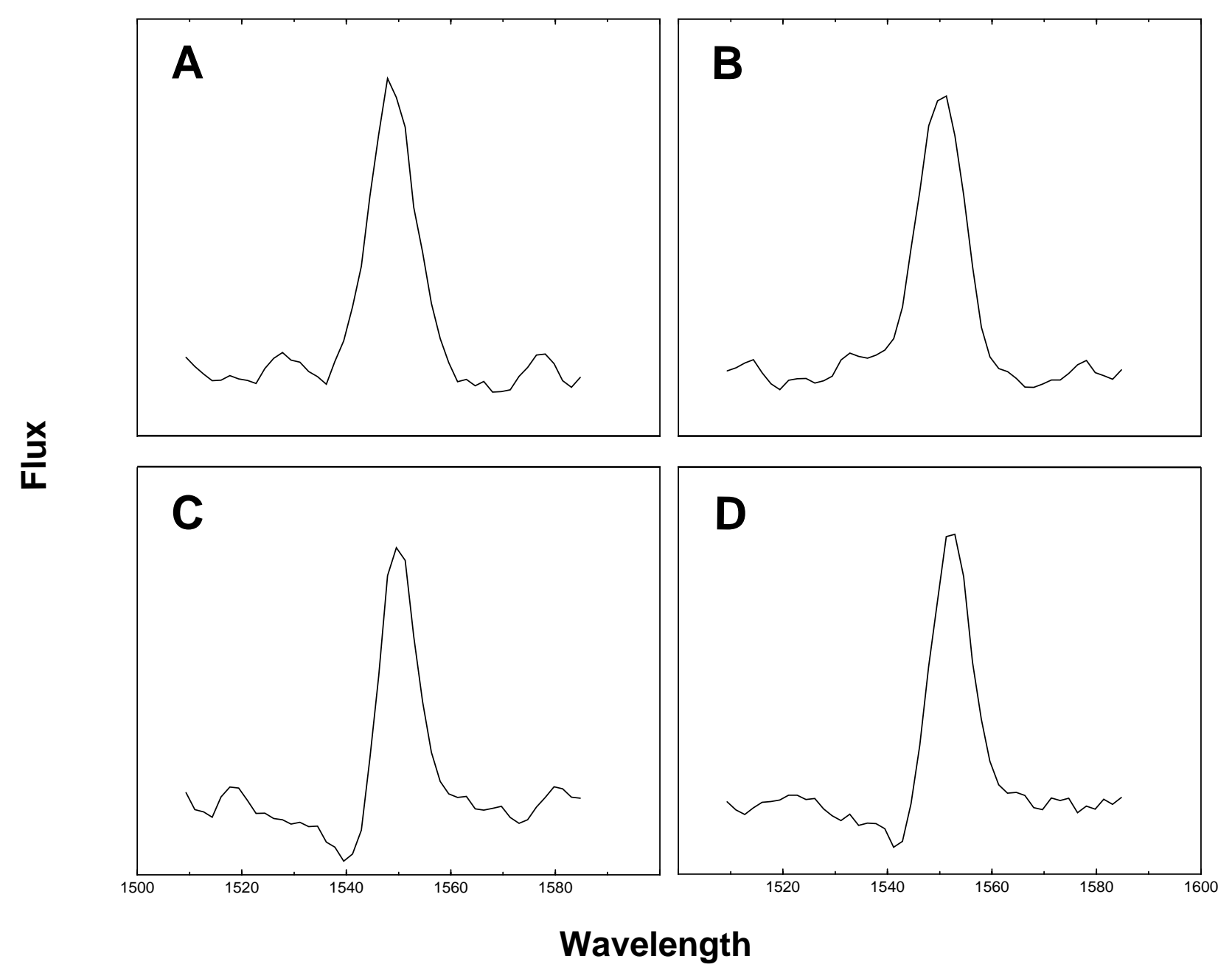

Fig. 15. CIV1550 line, at phases $\phi_{\mathrm{em}}=0.550,0.568,0.633,0.646$. Scales as in Fig. 14 .

of the modulation of the intensity, of order of 20 percent. It is difficult to explain by a pure aspect effect and particularly so at an inclination of $\sim 20^{\circ}$. Hence explanation of both the amplitude and irregularity of the modulation of the emission line, and its P-Cygni profile calls for an asymmetric wind of optical thickness varying between different lines of sight. In order to explain the coherent periodic modulation, the asymmetry must remain fixed over the whole interval of observations. We defer discussion of the rather stringent requirement imposed on any models by such an asymmetry to Sect. 6.7.

Similar but more noisy results are obtained for SiIV1393/1402 A doublet. They are presented in Figs. 18 and 19. In particular, the period also agrees within the errors with the orbital one.

After we finished our investigation, Prinja et al. (2000) presented the results of their analysis of the absorption line profiles in V603 Aql spectra, based on rapid Hubble Space Telescope ultraviolet spectroscopy. They reported the presence of episodic blue-shifted absorption (up to $2500 \mathrm{~km} \mathrm{~s}^{-1}$ ). During 5 hrs of observations (consisting of 4 observing blocks), they recorded three absorption events, each lasting 10-15 min. They concluded that the derived velocity, acceleration and optical depth properties provided a picture of stochastically variable structure in the outflow. They didn't find any cyclic or

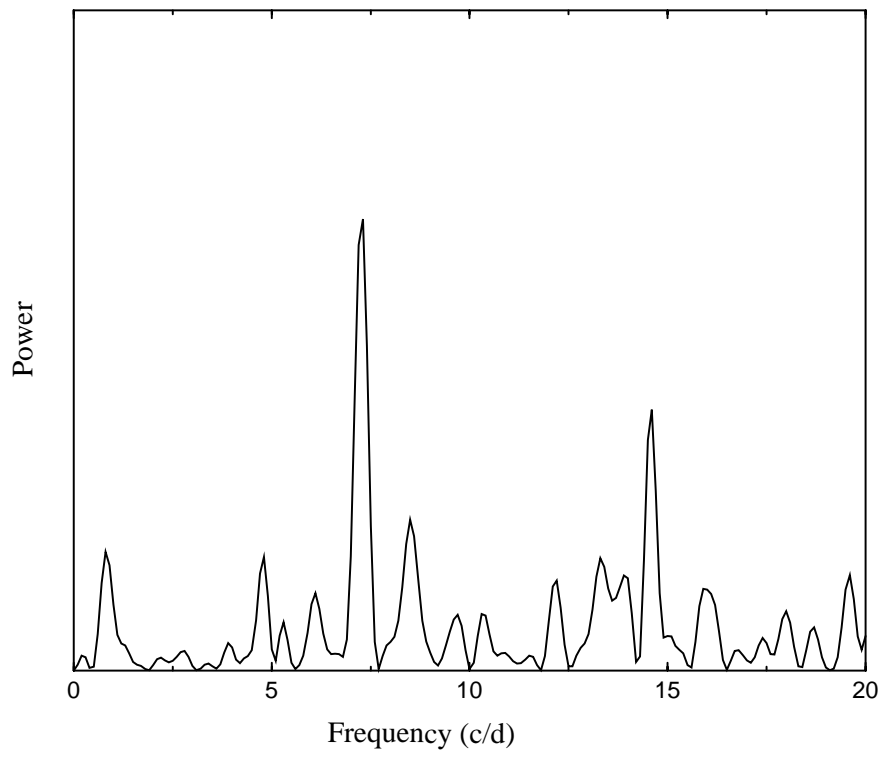

Fig. 16. Power spectrum of strength of the absorption component of CIV1550 line. $X$ axis is frequency in cycles per day.

modulated behavior in the overall absorption properties. They also didn't notice any significant time-dependent changes in the 
Table 3. Frequency of modulation for absorption and emission part of CIV1550 line.

\begin{tabular}{ccc}
\hline \hline & $f[\mathrm{c} / \mathrm{d}]$ & Epoch of max. \\
\hline Emission CIV & $7.246 \pm 0.023$ & $3.0677 \pm 0.0030$ \\
Absorption CIV & $7.244 \pm 0.050$ & $3.0383 \pm 0.0056$ \\
Average CIV & $7.246 \pm 0.020$ & - \\
SiIV & $7.206 \pm 0.056$ & $3.0654 \pm 0.0053$ \\
Orbital & $7.242 \pm 0.006^{a}$ & - \\
Jun. 3-8 & $7.468 \pm 0.030^{a}$ & - \\
Jun. 3-26 & $7.462 \pm 0.006^{a}$ & - \\
Jul. 24-Aug. 11 & $7.435 \pm 0.013^{a}$ & - \\
Aug. 8-Sep. 14 & $7.429 \pm 0.006^{a}$ & - \\
Average Phot. & $7.446 \pm 0.009$ & - \\
\hline
\end{tabular}

${ }^{a}$ From Patterson et al. (1997).

emission components. But, note that the time intervals used by the authors - due to peculiarities of the HST orbit - were much shorter then our IUE coverage and indeed shorter than the time scales of the modulations discussed by us. For this reason, it is not surprising that Prinja et al. (2000) missed the underlying regularity in the line modulation. The importance of their and other HST work lies in the demonstration of the dramatically short time scales of the spectral variations in V603 Aql.

\section{Discussion}

There is no doubt that all the non-stellar energy radiated by CVs comes from accretion. However, the role of different wavelengths in the exchange of energy within the binary is not so clear. Empirical investigations are hampered by the dearth of extended multiwavelength and truly simultaneous observations. Our data yield a unique opportunity to glimpse the energy flow between the sources of X-ray and UV continua.

\subsection{Energy transport by radiation}

On the one hand the results discussed in Sect. 4 indicate some correlation of continuum X-ray and UV fluctuations at zero lag, at least in the lightcurves binned at the rather long time scales exceeding $400 \mathrm{~s}$. If confirmed by further observations and with an improved time resolution, this result favors illumination as a possible means for the re-distribution of energy as corresponding lags are less than $1 \mathrm{~s}$. Although observations give no clue as to which energy form is primordial, theory favors X-ray illumination as the cause of UV flickering as the photon energy degradation occurs much easier than the reverse.

\subsection{Accretion power and lag in UV-X-ray correlation}

On the other hand modulation of the accretion energy sources between the hot spot and disk and/or the disk and its boundary layer would correspond to lags considerably over $15 \mathrm{~min}$. This time scale is predicted by the time-dependent models of the accretion by Meyer \& Meyer-Hoffmeister (1984) and Smak (1984), and confirmed by the multiwavelength observations

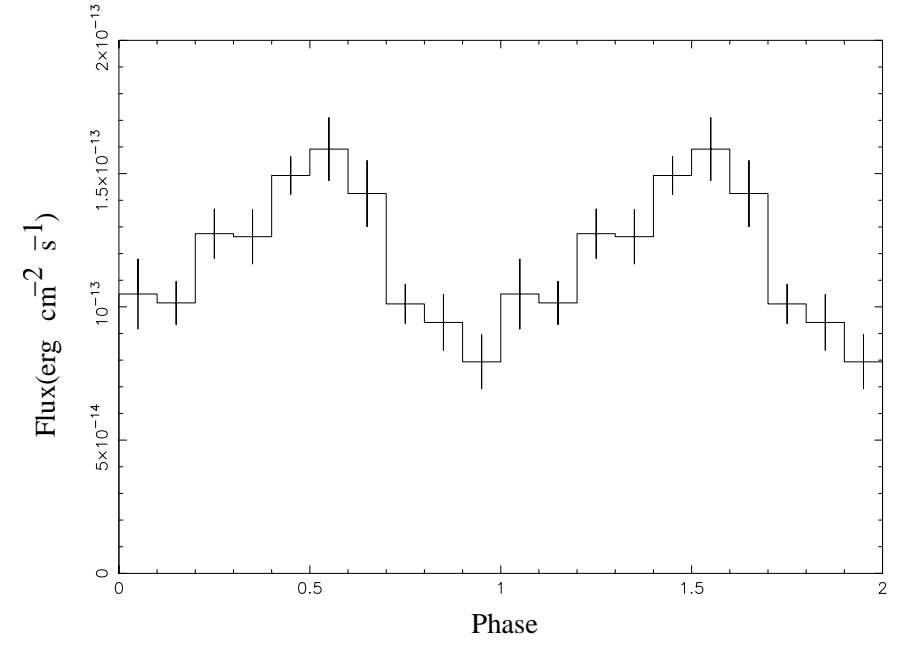

Fig. 17. Intensity of CIV1550 emission line phase, in erg $\mathrm{cm}^{-2} \mathrm{~s}^{-1} \AA^{-1}$, folded and binned with the orbital period. Phase 0 corresponds to HJD 2448903.1397. To explain this modulation at orbital inclination $i \sim 20$ we postulate absorption by an asymmetric wind.

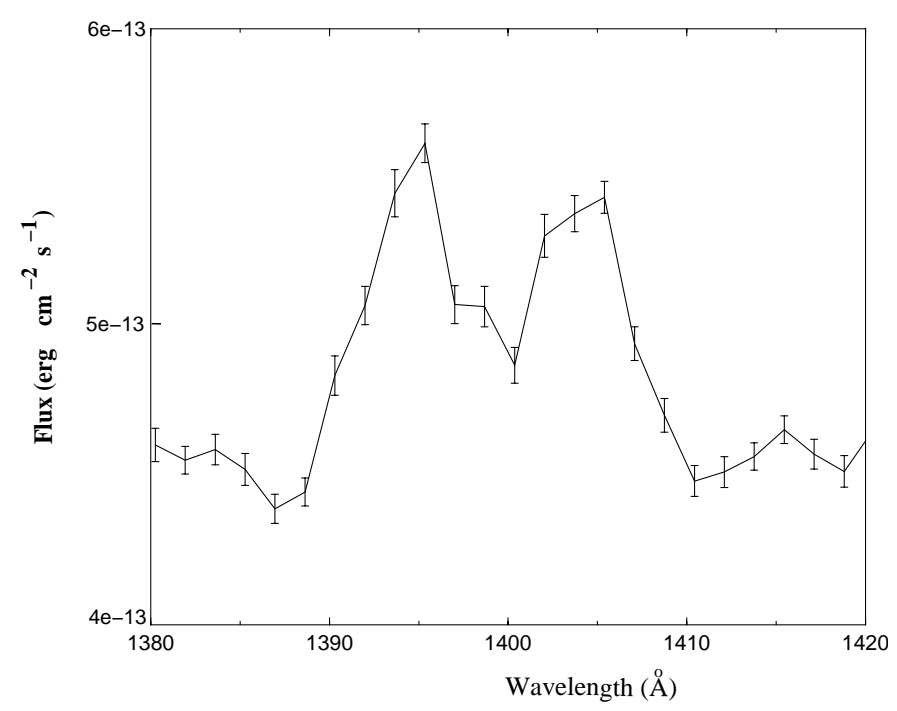

Fig. 18. Average profile of SiIV1393/1402 A doublet line, from 100 IUE spectra. Error bars indicate $\pm 1 \sigma$ range of the mean. Scatter of individual observations is 10 times more.

of the outbursts of dwarf novae (e.g. Schwarzenberg-Czerny et al. 1985). Our observations do not show noticeable correlation of UV and X-ray fluxes at lags significantly different from 0 . The only hint that some lagged correlation might be present is in the UV color and X-ray flux CCF discussed in Sect. 4.3. The evidence of the correlation is weak. If true, it would favor a scenario producing UV radiation as the primary effect of accretion, and X-rays as an after-effect. There are several effects possibly masking the lagged correlation in the UV and X-ray fluxes. We already mentioned the first cause involving the energy transport by illumination (Sect. 6.1).

The second cause may be purely observational. Our resolution in time and/or in intensity is limited, due to the finite UV exposure time and the demand for adequate X-ray statistics. We could not discriminate between 0 and 10 min lags in the 


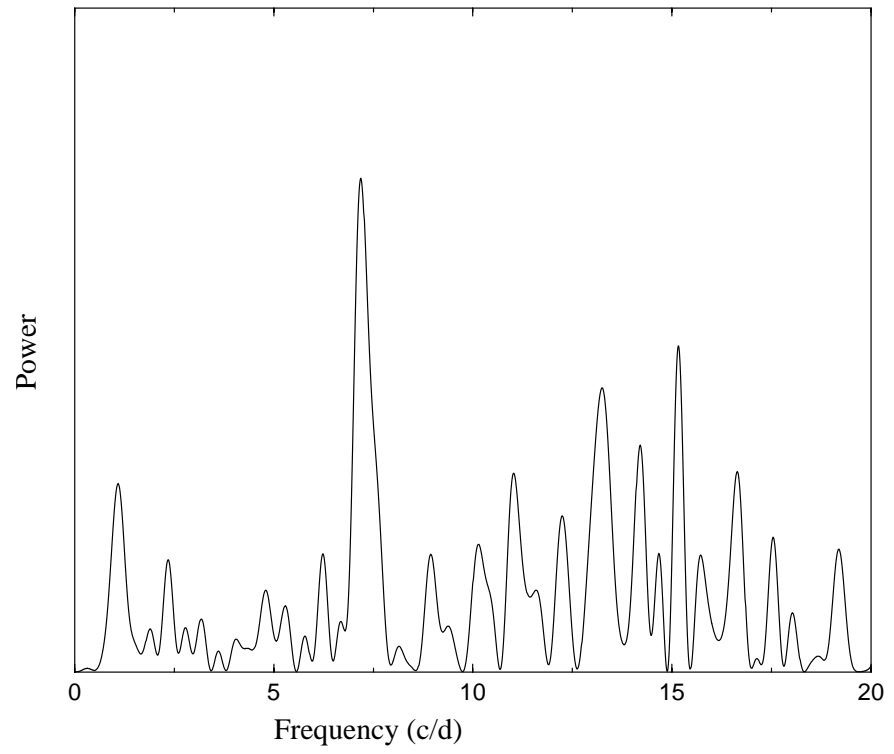

Fig. 19. Power spectrum of intensity of the SiIV1393/1402 A doublet emission line.

correlation. Note that the correlation at 0 lag is detected most reliably for bins as wide as $\pm 20 \mathrm{~min}$. The quality of UV calibration and $\mathrm{X}$-ray statistics at the adopted binning/exposure resolution yield noise amounting to over 5 percent of the signal. Hence our observations do not exclude the presence of a correlation with shorter lags and/or smaller amplitudes.

The third cause may concern the statistical properties of the accretion sources of radiation at different rings. Naively one would expect cooler radiation modulated at longer time scales of the flow from the outer regions of the accretion disk. With due time lag for mass flow inwards this modulation should affect the accretion rate in the inner regions of the disk. If the UV and X-rays originate predominantly from different regions, such a lagged correlation should be observed. However, the inner rings of the disk cause intrinsic variability at still higher frequencies, with a cut off at the local thermal time scale. A sufficiently steep rise of power at high frequencies may cause enough drift of power to lower frequencies to mask any accretion driven modulation.

Clearly, our observations call for a more thorough review of the time effects consistent with the current accretion disk model. However, it would be premature to imply serious inconsistency of our observations with the accepted accretion model without prior thorough analysis of the listed thee alternative explanations.

\subsection{Sources of the wind}

The stream and orbital velocities and their radial components are small compared to the width of the line features, exceeding $w \sim 1000 \mathrm{~km} \mathrm{~s}^{-1}$. For this reason, the hot spot does not seem a likely place for excitation of the wind (Rozyczka \& Schwarzenberg-Czerny 1987). The sole remaining source of the high-velocity wind remains the inner parts of the accretion disk and the vicinity of the white dwarf itself. Indeed, for a white dwarf mass exceeding $0.5 M_{\odot}$ the near-surface Kepler and escape velocities amount to at least 2700 and $3800 \mathrm{~km} \mathrm{~s}^{-1}$. These high velocities are observed by us and by Prinja et al. (2000). So, the explanation of line features corresponding to a wind velocity of order $w$ poses no problem, even after allowance is made for a factor of 2 for projection effects.

The keplerian period at the inner disc radius constitutes a fraction of a minute. This time scale corresponds to the fastest variability time scale ( $<1 \mathrm{~min}$ ) registered by Prinja et al. (2000). Summarizing, both lines of reasoning favor the vicinity of the white dwarf as the source of the wind. Popular scenarios of wind excitation involve radiation pressure (e.g. Proga 1999 and references there).

\subsection{Modulation of the P Cygni line}

We observe a modulation of the P-Cyg emission and absorption components with the orbital period. The modulation of the absorption component must be tied to the variable density in the line of sight, due to the variable aspect and/or ejection of gas bubbles. A smooth variation would favor aspect while a sharp variation may be caused by an inhomogeneous gas flow. Our observations provide no clear clues in this respect. However, Prinja et al. (2000) observed a modulation of the absorption component decomposed into separate events lasting about $15 \mathrm{~min}$ and variable on a time scale as short as $1 \mathrm{~min}$. This kind of modulation would favor inhomogeneous flow.

Some chromospheric emission on top of the photospheric absorption might be present in the Ly- $\alpha$ line but it is masked by the geocoronal emission. However, such effects are not expected in such a high excitation line as CIV1550 ̊. Generally, P-Cygni profiles are explained by self-absorption in the accelerating and cooling wind (Castor et al. 1975; Proga et al. 1998).

\subsection{Symmetry considerations}

An explanation of the coherent modulation of the lines is prone to difficulties. The nebular expansion of V603 Aql provided a textbook example of the successful reconstruction of the geometry from an analysis of the radial velocities of the ejecta, yielding an orbital inclination $\sim 20^{\circ}$ (Mustel \& Boyarchuk 1970; Weaver 1974). This estimate agrees with the small orbital velocity, which gives an inclination $i \sim 17^{\circ}$ (Warner 1995) and with the lack of eclipses and, indeed, lack of any traces of orbital modulation in extensive X-ray, UV continuum and visual range lightcurves. At this low inclination, eclipses and aspect effects are negligible, so that a coherent modulation of the emission lines is difficult to explain.

In cataclysmic binaries white dwarfs come in two brands: magnetic and non-magnetic. The magnetic white dwarfs often manifest themselves by polarization of their radiation and/or by pulsed radiation due to the light-house effect. X-ray observations are most sensitive in a search for magnetic white dwarfs. Thus in the present context, lack of any X-ray modulation argues against a magnetic white dwarf in V603 Aql. An exactly on-axis and/or isotropically radiating magnetic pole does not seem a likely configuration deserving further consideration. For a non-magnetic white dwarf there are no obvious 
reasons to break the axial symmetry of the disk-star interface powering the wind. Because of viscosity and negligible tidal action, the inner disk should be perfectly circularized. By symmetry arguments the boundary layer, radiation field and acceleration of the wind should also remain axially symmetric. It is difficult to find other external reasons for breaking the axial symmetry of the highly supersonic wind.

\subsection{Asymmetric wind}

Difficulties with aspect effects, the amplitude and irregularity of the modulation and the presence of P-Cygni profiles all indicate that an explanation of the observed effects should involve the absorbing wind. The absorption in the wind must be varying between different lines of sight in a non-axisymmetric fashion. However, in order to explain the coherent periodic modulation, the asymmetry should last over the whole interval of observations.

Let us assume that the intensity of an emitted line depends solely on the cosine $\mu$ of the angle between the directions from the disc center towards the observer and the secondary star, $I(\mu)=I_{0}+I_{1} \mu$. Writing explicitly in terms of the orbital phase $\phi$ one obtains

$I(\phi)=I_{0}+I_{1} \cos \phi \sin i$

where $i$ denotes orbital inclination. For the inclination $i \approx 17^{\circ}$ and the amplitude of modulation $\max \left|I(\phi)-I_{0}\right| / I_{0}=0.19$ the required anisotropy is $I_{1} / I_{0}=0.19 / \sin i=0.65$. This amount of asymmetry of the wind would have to occur either by a yet unidentified asymmetry of the inner disc or by a kind of spontaneous symmetry breaking within the wind itself.

\subsection{Extent of the wind and cause of its asymmetry}

A unique event of spontaneous breaking of the symmetry by a kind of hydrodynamic instability does not solve the difficulty. In order to explain a modulation of the absorption coherent over $\tau \sim 3$ days, the asymmetry must be preserved over the same time interval. However, the distance covered by the wind during such an interval reaches $w \tau \sim 10^{13} \mathrm{~cm}$, i.e. a hundred times the size of the binary. To produce the absorption component the envelope would have to still remain opaque in the lines at this large distance. Such a potent wind may be feasible during nova explosions but certainly not during our observations in quiescence. Permanent asymmetry in the source of the wind appears as a more likely alternative. In view of the lack of evidence for a strong magnetic field, the cause of such an asymmetry remains mysterious. Perhaps some residual weak magnetic field?

\section{Conclusions}

The first analysis of a continuous long series of UV and X-ray simultaneous observations of V603 Aql has been performed. Our results show that the Lucy-Richardson deconvolution algorithm can be successfully used for the extraction of the UV spectra from multiply exposed IUE frames. Our tests also suggest that this method leads to better results than the older algorithm based on the classic Gaussian fit.
The absence of any periodic modulation of the X-rays lends no support to a classification of V603 Aql as an intermediate polar.

Comparing the old US89 results with the results from our new observations, we noticed that the behavior of the star changed in 3 yrs. The short period modulation, which US89 found in the 1989 UV continuum lightcurve and which was recovered in the same data re-reduced with our new software, is undetectable in the new data. Our discovery of a strong modulation in the CIV1550 emission line intensities, with a period consistent with the orbital period, and the absence of such a modulation in the UV continuum seem to suggest that the UV continuum and emission lines are being produced in different parts of the accretion disk.

Our observations hint at a correlation between the X-ray and UV emission at nearly zero lag. This leads us to speculate that the underlying mechanism of UV flickering is X-ray illumination.

The reason behind the modulation in the emission and absorption components of the spectral lines is still not clear. As we showed earlier, both parts of the P-Cygni profile are strictly correlated with the phase of orbital motion. One possible explanation may be that the source of emission is the external, hot chromosphere of the disk, while the absorption is created in a colder and asymmetrical stellar wind.

P-Cygni profiles with variations in the spectral line intensities in the visual band were noticed by Ringwald \& Naylor (1998) in the case of BZ Camelopardalis. But it's not obvious whether the mechanism of a cold stellar wind causing this effect in BZ Cam is similar to the hot, highly excited wind in V603 Aql.

Acknowledgements. This paper reports observations made possible thanks to a stipend of the Max-Planck-Geselschaft to ASC and hospitality of the Max-Planck Institut fuer Extraterrestische Physik in Garching. We thank Wolfgang Pietsch for his expertise and interest in the present project and to F. Piche for communication of the optical observations. We are grateful to J. Smak for discussions and for numerous detailed comments. The presentation of our paper benefited from the critical yet thoughtful comments by the anonymous referee. We acknowledge funding of our research by NASA grant NAG 5-2315 to PS and by KBN grants 2P03D01818 (ASC), 5P03D00220 (WB).

\section{References}

Arenas, J., Catalán, M. S., Augusteijn, T., \& Retter, A. 2000, MNRAS, 311,135

Borczyk, W. 1999, Ph.D. Thesis, Nicolaus Copernicus University, Torun, Poland (in Polish)

Castor, J. I., Abbott, D. C., \& Klein, R. I. 1975, ApJ, 195, 157

Edelson, R. A., \& Krolik, J. H. 1988, ApJ, 333, 646

Drechsel, H., Rahe, J., \& Holm, A. 1981, A\&A, 99, 166

Fisz, M. 1963, Probability Theory and Mathematical Statistics (New York: Wiley), 536

Friedjung, M., Selvelli, P., \& Casatella, A. 1997, A\&A, 318, 204

Haefner, R., \& Metz, K. 1985, A\&A, 145, 311

Kraft, R. P. 1964, A\&A, 139, 457

Lucy, L. B. 1974, AJ, 79, 745 
Mauche, C. W., Brickhouse, N., Howell, S., et al. 2000, American Astronomical Society, Head meeting \#32, \#45.06

Meyer, F., \& Meyer-Hoffmeister, E. 1984, A\&A, 132, 143

Mustel, E. R., \& Boyarchuk, A. A. 1970, Ap\&SS, 6, 183

Patterson, J., Thomas, G., \& Skillman, D. 1993, ApJS, 86, 235

Patterson, J., Kemp, J., Saad, J., et al. 1997, PASP, 109, 468

Patterson, J., \& Raymond, J. C. 1985, ApJ, 292, 535

Prinja, R. K., Knigge, Ch., Ringwald, F. A., \& Wade, R. 2000, MNRAS, 318, 368P

Prinja, R. K., \& Rosen, S. 1993, MNRAS, 262, L37

Proga, D. 1999, MNRAS, 304, 938

Proga, D., Stone, J. M., \& Drew, J. E. 1998, MNRAS, 295, 595

Ringwald, F. A., \& Naylor, T. 1998, ApJ, 115, 286

Rozyczka, M., \& Schwarzenberg-Czerny, A. 1987, Acta Astron., 37, 2

Schwarzenberg-Czerny, A. Udalski, A., \& Monier, R. 1992, ApJ, 401, L19

Schwarzenberg-Czerny, A., Ward, M., Hanes, D. A., et al. 1985, MNRAS, 212, 645

Schwarzenberg-Czerny, A. 1989, MNRAS, 241, 153
Schwarzenberg-Czerny, A. 1991, MNRAS, 253, 198

Schwarzenberg-Czerny, A. 1993, Interactive Data Handling Program ULA, users manual

Schwarzenberg-Czerny, A. 1996, ApJ, 460, L107

Seitter, W. C. 1990, IAU Coll., 122, 79

Selvelli, P. L., Gilmozzi, R., \& Friedjung, M. 1998, Ultraviolet Astrophysics, Beyond the IUE Final Archive Proc. Conf.

Smak, J. 1984, Acta Astron., 34, 161

Spruit, H. C. 1994, A\&A, 289, 441

Szkody, P., \& Mateo, M. 1986, ApJ, 301, 286

Trumper, J. 1983, Adv. Space Res., 2, 241

Udalski, A., \& Schwarzenberg-Czerny, A. 1989, Acta Astron., 39, 125

Voges, W. 1992, Space Science, Proc. ISY Conf. ESA ISY-3, ESA Publications, 9

Weaver, H. 1974, Highlight of Astr., 3, 509

Warner, B. 1995, Cataclysmic Variable Stars (Cambridge: Cambridge University Press)

Zimmermann, H.-U., Belloni, T., Boese, G., et al. 1992, in Data Analysis in Astronomy IV, ed. V. Di Gesu, L. Scarsi, R. Buccheri, et al. (New York and London: Plenum Press), 141 